\title{
REGULAR VERSUS SINGULAR SOLUTIONS IN A QUASILINEAR INDEFINITE PROBLEM WITH AN ASYMPTOTICALLY LINEAR POTENTIAL
}

\author{
JULIAN LÓPEZ-GÓMEZ AND PIERPAOLO OMARI
}

ABStRACT. The aim of this paper is analyzing the positive solutions of the quasilinear problem

$$
-\left(u^{\prime} / \sqrt{1+\left(u^{\prime}\right)^{2}}\right)^{\prime}=\lambda a(x) f(u) \text { in }(0,1), \quad u^{\prime}(0)=0, u^{\prime}(1)=0,
$$

where $\lambda \in \mathbb{R}$ is a parameter, $a \in L^{\infty}(0,1)$ changes sign once in $(0,1)$ and satisfies $\int_{0}^{1} a(x) d x<0$, and $f \in \mathcal{C}^{1}(\mathbb{R})$ is positive and increasing in $(0,+\infty)$ with a potential, $F(s)=\int_{0}^{s} f(t) d t$, quadratic at zero and linear at $+\infty$. The main result of this paper establishes that this problem possesses a component of positive bounded variation solutions, $\mathscr{C}_{\lambda_{0}}^{+}$, bifurcating from $(\lambda, 0)$ at some $\lambda_{0}>0$ and from $(\lambda, \infty)$ at some $\lambda_{\infty}>0$. It also establishes that $\mathscr{C}_{\lambda_{0}}^{+}$consists of regular solutions, if, and only if,

$$
\int_{0}^{z}\left(\int_{x}^{z} a(t) d t\right)^{-\frac{1}{2}} d x=+\infty, \quad \text { or } \quad \int_{z}^{1}\left(\int_{x}^{z} a(t) d t\right)^{-\frac{1}{2}} d x=+\infty .
$$

Equivalently, the small positive regular solutions of $\mathscr{C}_{\lambda_{0}}^{+}$become singular as they are sufficiently large if, and only if,

$$
\left(\int_{x}^{z} a(t) d t\right)^{-\frac{1}{2}} \in L^{1}(0, z) \quad \text { and } \quad\left(\int_{x}^{z} a(t) d t\right)^{-\frac{1}{2}} \in L^{1}(z, 1) .
$$

This is achieved by providing a very sharp description of the asymptotic profile, as $\lambda \rightarrow \lambda_{\infty}$, of the solutions. According to the mutual positions of $\lambda_{0}$ and $\lambda_{\infty}$, as well as the bifurcation direction, the occurrence of multiple solutions can also be detected.

\section{INTRODUCTION}

This paper analyzes the quasilinear indefinite Neumann problem

$$
\left\{\begin{array}{l}
-\left(\frac{u^{\prime}}{\sqrt{1+\left(u^{\prime}\right)^{2}}}\right)^{\prime}=\lambda a(x) f(u) \quad \text { in }(0,1), \\
u^{\prime}(0)=u^{\prime}(1)=0
\end{array}\right.
$$

which is a one-dimensional prototype of

$$
\left\{\begin{array}{l}
-\operatorname{div}\left(\frac{\nabla u}{\sqrt{1+|\nabla u|^{2}}}\right)=g(x, u) \text { in } \Omega, \\
-\frac{\nabla u \cdot \nu}{\sqrt{1+|\nabla u|^{2}}}=\sigma \quad \text { on } \partial \Omega,
\end{array}\right.
$$

where $\Omega$ is a bounded regular domain of $\mathbb{R}^{N}$ with outward pointing normal $\nu$, and $g: \Omega \times \mathbb{R} \rightarrow \mathbb{R}$ and $\sigma: \partial \Omega \rightarrow \mathbb{R}$ are given functions. Problems involving the mean curvature operator play a pivotal role in the mathematical analysis of a number of geometrical and physical issues, such as prescribed mean curvature problems for cartesian surfaces in the Euclidean space $[29,3,22,30,9,17,15,18,16]$, capillarity phenomena

Date: March 6, 2020.

2010 Mathematics Subject Classification. Primary: 35J93, 34B18. Secondary: 35J15, 35B09.

Key words and phrases. Quasilinear problem, mean curvature operator, Neumann boundary condition, indefinite weight, classical solution, bounded variation solution, positive solution, regular solution, formation of singularities, asymptotic profile.

J. López-Gómez has been supported by the Research Grant PGC2018-097104-B-100 of the Ministry of Science, Technology and Universities of Spain and by the Institute of Inter-disciplinar Mathematics of Complutense University. P. Omari has been supported by "Università degli Studi di Trieste-Finanziamento di Ateneo per Progetti di Ricerca Scientifica-FRA 2018". This paper has been written under the auspices of INdAM-GNAMPA. 
for compressible or incompressible fluids [5, 11, 10, 19, 20, 12, 13, 14, 7, 6], and reaction-diffusion processes where the flux features saturation at high regimes $[28,21,4]$.

When dealing with problem (1.1), $\lambda \in \mathbb{R}$ is viewed as a parameter and the following assumptions will be considered eventually:

$\left(\mathrm{a}_{1}\right) a \in L^{\infty}(0,1)$ satisfies

$$
\int_{0}^{1} a(x) d x<0
$$

and there exists a point $z \in(0,1)$ such that $a(x)>0$ a.e. in $(0, z)$ and $a(x)<0$ a.e. in $(z, 1)$;

$\left(\mathrm{a}_{2}\right) \int_{0}^{z}\left(\int_{x}^{z} a(t) d t\right)^{-\frac{1}{2}} d x=+\infty, \quad$ or $\quad \int_{z}^{1}\left(\int_{x}^{z} a(t) d t\right)^{-\frac{1}{2}} d x=+\infty$;

$\left(\mathrm{f}_{1}\right) f \in \mathcal{C}^{1}(\mathbb{R})$ is strictly increasing and, for some constant $h>0$, satisfies

$$
\lim _{s \rightarrow 0^{+}} \frac{f(s)}{s}=1 \quad \text { and } \quad \lim _{s \rightarrow+\infty} f(s)=h .
$$

As the function $\left(\int_{x}^{z} a(t) d t\right)^{-\frac{1}{2}}$ is continuous and positive in $x \in[0,1] \backslash\{z\}$, the integrals

$$
\int_{0}^{z}\left(\int_{x}^{z} a(t) d t\right)^{-\frac{1}{2}} d x \text { and } \int_{z}^{1}\left(\int_{x}^{z} a(t) d t\right)^{-\frac{1}{2}} d x
$$

are well-defined and each of them is either finite, or it equals $+\infty$. Condition $\left(\mathrm{a}_{2}\right)$ requires some of them, or both, to be divergent.

Condition $\left(\mathrm{f}_{1}\right)$ entails that $f(0)=0$ and $f(s)>0$ for all $s>0$. Moreover, the associated potential,

$$
F(s)=\int_{0}^{s} f(t) d t
$$

is quadratic at 0 and linear at $+\infty$. Some paradigmatic examples of $f$ satisfying $\left(\mathrm{f}_{1}\right)$, for a given $h>0$, are provided by

$$
f(s)=\frac{h s}{\sqrt{h^{2}+s^{2}}} \text { and } f(s)=h \tanh \left(\frac{s}{h}\right) .
$$

Throughout this paper, we are going to use the next notions of solution.

- A couple $(\lambda, u)$ is said to be a regular solution of (1.1) if $u \in W^{2,1}(0,1)$ and it satisfies the differential equation a.e. in $(0,1)$, as well as the boundary conditions.

- A couple $(\lambda, u)$ is said to be a bounded variation solution, or shortly BV-solution, of (1.1) if $u \in B V(0,1)$ and it satisfies

$$
\int_{0}^{1} \frac{D^{a} u D^{a} \phi}{\sqrt{1+\left|D^{a} u\right|^{2}}} d x+\int_{0}^{1} \frac{D^{s} u}{\left|D^{s} u\right|} D^{s} \phi=\int_{0}^{1} \lambda a f(u) \phi d x
$$

for all $\phi \in B V(0,1)$ such that $\left|D^{s} \phi\right|$ is absolutely continuous with respect to $\left|D^{s} u\right|$ (cf. [2]).

- A couple $(\lambda, u)$ is said to be a singular solution of (1.1) if it is a bounded variation solution of (1.1) such that $u \in B V(0,1) \backslash W^{2,1}(0,1)$.

- Once the pair $(\lambda, u)$ solves (1.1) in any of the previous senses, it is said that $(\lambda, u)$ is a positive solution if, in addition,

$$
\lambda \geq 0 \text { and } \operatorname{ess} \inf u>0 .
$$

As usual, for any function $v \in B V(0,1)$,

$$
D v=D^{a} v d x+D^{s} v
$$

stands for the Lebesgue decomposition of the Radon measure $D v$ and $\frac{D^{s} v}{\left|D^{s} v\right|}$ denotes the density function of the measure $D^{s} v$ with respect to its total variation $\left|D^{s} v\right|$ (cf. [1]).

By $\left(\mathrm{a}_{1}\right)$, any regular solution, $(\lambda, u)$, of $(1.1)$, with $\lambda>0$, satisfies $u^{\prime}(x)<0$ for every $x \in(0,1)$, $u^{\prime}(0)=u^{\prime}(1)=0$, and, since $u$ is concave on $[0, z)$ and convex on $(z, 1]$,

$$
-u^{\prime}(z)=\left\|u^{\prime}\right\|_{L^{\infty}(0,1)} .
$$


Moreover, by [24, Prop. 3.6], any positive singular solution, $(\lambda, u)$, of (1.1), with $\lambda>0$, satisfies

$$
\begin{aligned}
&\left.u\right|_{[0, z)} \in W_{\mathrm{loc}}^{2,1}[0, z) \cap W^{1,1}(0, z) \text { and is concave, } \\
&\left.u\right|_{(z, 1]} \in W_{\mathrm{loc}}^{2,1}(z, 1] \cap W^{1,1}(z, 1) \text { and is convex, } \\
& u^{\prime}(x)<0 \text { for every } x \in(0,1) \backslash\{z\}, u^{\prime}(0)=u^{\prime}(1)=0 \text { and } \\
& u^{\prime}\left(z^{-}\right)=u^{\prime}\left(z^{+}\right)=-\infty,
\end{aligned}
$$

where $u^{\prime}\left(z^{-}\right)$and $u^{\prime}\left(z^{+}\right)$are the left and the right Dini derivatives of $u$ at $z$. The left and the right limits of $u$ at $z$ will be respectively denoted by $u\left(z^{-}\right)$and $u\left(z^{+}\right)$. According to (1.4), throughout this paper we set

$$
\frac{-u^{\prime}(z)}{\sqrt{1+\left(u^{\prime}(z)\right)^{2}}}=1 \text {. }
$$

Any solution, $(\lambda, u)$, of (1.1) with $\lambda \geq 0$ and $u(x) \geq 0$ for a.e. $x \in(0,1)$ satisfies either $u=0$, or ess $\inf u>0$. Indeed, if $\lambda=0$, then $u$ is necessarily constant (see, e.g., [24, Sect. 3]), whereas in case $\lambda>0$ the conclusion easily follows combining (1.3) with the uniqueness of solution to the Cauchy problem associated with the differential equation of (1.1). Moreover, it follows from [26, Prop. 1.1] that $\lambda \geq 0$ is necessary for the existence of a bounded variation solution, either regular or singular, satisfying ess inf $u>0$. In particular, $\lambda>0$ is necessary for the existence of non-constant positive solutions.

Under the assumption $\left(\mathrm{a}_{1}\right)$, the linear eigenvalue problem

$$
\left\{\begin{array}{l}
-\varphi^{\prime \prime}=\lambda a(x) \varphi \quad x \in(0,1) \\
\varphi^{\prime}(0)=\varphi^{\prime}(1)=0 .
\end{array}\right.
$$

possesses two principal eigenvalues: $\lambda=0$ and $\lambda=\lambda_{0}>0$. By a principal eigenvalue, it is meant a value of $\lambda$ for which (1.5) admits a positive eigenfunction, $\varphi$. Moreover, these eigenvalues are algebraically simple and the associated eigenfunctions, 1 and $\varphi_{0}$, are positive and separated away from zero; a recent synthesis of the available results on this topic is contained in [23, Ch. 9].

Throughout this paper, we will denote by $\mathscr{S}^{+}$the set of the positive bounded variation solutions of (1.1) closed by adding $(0,0)$ and $\left(\lambda_{0}, 0\right)$, which are the unique possible bifurcation points to positive solutions from the trivial solution branch $(\lambda, u)=(\lambda, 0)$, i.e.,

$$
\mathscr{S}^{+}=\{(\lambda, u) \mid(\lambda, u) \text { is a positive BV-solution of }(1.1)\} \cup\left\{(0,0),\left(\lambda_{0}, 0\right)\right\} \subset \mathbb{R} \times B V(0,1) .
$$

The set $\mathscr{S}^{+}$is endowed with the topology of the strict convergence of $\mathbb{R} \times B V(0,1)$. Namely, if $\left(\left(\lambda_{n}, u_{n}\right)\right)_{n \geq 1}$ is a sequence in $\mathscr{S}^{+}$and $(\lambda, u) \in \mathscr{S}^{+}$, it is said that

$$
\lim _{n \rightarrow+\infty}\left(\lambda_{n}, u_{n}\right)=(\lambda, u)
$$

whenever

$$
\lim _{n \rightarrow+\infty}\left(\left|\lambda_{n}-\lambda\right|+\left\|u_{n}-u\right\|_{L^{1}}+\left|\int_{0}^{1}\right| D u_{n}\left|-\int_{0}^{1}\right| D u||\right)=0 .
$$

The model (1.1) has been recently analyzed by the authors in [26], [27], [24] and [25]. In [26] the existence of bounded variation solutions was investigated by means of the methods of the calculus of variations and in [27] the existence of regular solutions was dealt with by means of phase plane analysis and bifurcation techniques. The main result of [24] established the existence of a component of bounded variation solutions bifurcating from the trivial solution $(\lambda, 0)$ when the associated potential of $f, F$, is quadratic at 0 . Finally, assuming that $F$ is super-quadratic at 0 and superlinear at $+\infty$, the main result of [25] characterized whether the solutions of (1.1) for sufficiently small $\lambda>0$ are regular, or singular, in terms of the integrability properties of the weight function $a$.

Here, our attention focuses on the case where $F$ is quadratic at 0 and linear at $+\infty$; our main aim being to discuss the fine structure of the set $\mathscr{S}^{+}$of positive solutions of (1.1), in particular, establishing the existence of a component bifurcating from $(\lambda, 0)$ at some $\lambda_{0}>0$ and from $(\lambda, \infty)$ at some $\lambda_{\infty}>0$, as well as characterizing the formation of singularities and describing the precise asymptotic profile of the solutions with large norm: Figure 1 provides us with a picture of such profiles. Precisely, the main findings of this paper can be summarized as follows. 
Theorem 1.1. Assume $\left(\mathrm{a}_{1}\right)$ and $\left(\mathrm{f}_{1}\right)$. Then, the set $\mathscr{S}^{+}$contains, at least, two connected components, $\mathscr{C}_{0}^{+}$ and $\mathscr{C}_{\lambda_{0}}^{+}$, which satisfy the following conditions:

\section{(Bifurcation from 0 and from $\infty$ )}

(a) $\mathscr{C}_{0}^{+}=\{0\} \times[0,+\infty)$;

(b) $\mathscr{C}_{\lambda_{0}}^{+} \cap(\mathbb{R} \times\{0\})=\left(\lambda_{0}, 0\right)$;

(c) there exist $\lambda_{*}, \lambda^{*} \in(0,+\infty)$ such that $\lambda_{*}<\lambda_{0}<\lambda^{*}$ and

$$
\operatorname{proj}_{\mathbb{R}}\left(\mathscr{S}^{+} \backslash \mathscr{C}_{0}^{+}\right) \subseteq\left[\lambda_{*}, \lambda^{*}\right]
$$

thus, in particular, the component $\mathscr{C}_{\lambda_{0}}^{+}$is bounded in the parameter $\lambda \in \mathbb{R}$;

(d) $\operatorname{proj}_{L^{\infty}(0,1)} \mathscr{C}_{\lambda_{0}}^{+}$is unbounded;

(e) for every sequence in $\mathscr{S}^{+} \backslash \mathscr{C}_{0}^{+},\left(\left(\lambda_{n}, u_{n}\right)\right)_{n \geq 1}$, such that

$$
\lim _{n \rightarrow+\infty}\left\|u_{n}\right\|_{L^{\infty}}=+\infty
$$

necessarily

$$
\lim _{n \rightarrow+\infty} \lambda_{n}=\lambda_{\infty}=\left(h \int_{0}^{z} a(x) d x\right)^{-1} ;
$$

thus, $\lambda=0$ and $\lambda=\lambda_{\infty}$ are the unique values of $\lambda$ for which bifurcation from infinity of positive bounded variation solutions occurs;

\section{(Asymptotic profile)}

(f) for every sequence in $\mathscr{S}^{+} \backslash \mathscr{C}_{0}^{+},\left(\left(\lambda_{n}, u_{n}\right)\right)_{n \geq 1}$, such that

$$
\lim _{n \rightarrow+\infty}\left\|u_{n}\right\|_{L^{\infty}}=+\infty
$$

necessarily,

$$
\lim _{n \rightarrow+\infty} u_{n}(x)=+\infty
$$

and

$$
\lim _{n \rightarrow+\infty} u_{n}^{\prime}(x)=\frac{-\int_{0}^{x} a(t) d t}{\sqrt{\left(\int_{0}^{z} a(t) d t\right)^{2}-\left(\int_{0}^{x} a(t) d t\right)^{2}}},
$$

for all $x \in[0, z)$, while, for every $x \in(z, 1]$,

$$
\lim _{n \rightarrow+\infty} u_{n}(x)=u_{\infty}(x)
$$

where $u_{\infty} \in W_{\mathrm{loc}}^{2, \infty}(z, 1]$ solves the problem

$$
\left\{\begin{array}{l}
-\left(\frac{u^{\prime}}{\sqrt{1+\left(u^{\prime}\right)^{2}}}\right)^{\prime}=\lambda_{\infty} a(x) f(u) \quad \text { in }(z, 1), \\
u^{\prime}\left(z^{+}\right)=-\infty, \quad u^{\prime}(1)=0,
\end{array}\right.
$$

with $u_{\infty}(x)>0$ for all $x \in(z, 1]$ and either

or

$$
u_{\infty}\left(z^{+}\right)<+\infty \text { if } \int_{z}^{1}\left(\int_{x}^{z} a(t) d t\right)^{-\frac{1}{2}} d x<+\infty,
$$

$$
u_{\infty}\left(z^{+}\right)=+\infty \quad \text { if } \int_{z}^{1}\left(\int_{x}^{z} a(t) d t\right)^{-\frac{1}{2}} d x=+\infty
$$

\section{(Regularity versus development of singularities)}

(g) there exists a neighborhood, $\mathcal{U}$, of $\left(\lambda_{0}, 0\right)$ in $\mathbb{R} \times L^{\infty}(0,1)$ such that $\mathscr{C}_{\lambda_{0}}^{+} \cap \mathcal{U}$ consists of regular solutions;

(h) the set $\mathscr{S}^{+}$and, in particular, the component $\mathscr{C}_{\lambda_{0}}^{+}$consist only of regular solutions if, and only if, $\left(\mathrm{a}_{2}\right)$ holds; 
(i) if $\left(\mathrm{a}_{2}\right)$ fails, then all solutions $(\lambda, u)$ in $\mathscr{S}^{+}$and, in particular, in $\mathscr{C}_{\lambda_{0}}^{+}$, with $\lambda>0$ and sufficiently large $\|u\|_{L^{\infty}}$, are singular.

In particular, under conditions $\left(\mathrm{a}_{1}\right)$ and $\left(\mathrm{f}_{1}\right)$, the failure of $\left(\mathrm{a}_{2}\right)$ characterizes the development of singularities by the positive solutions of (1.1) along the component $\mathscr{C}_{\lambda_{0}}^{+}$. A simple example for which $\left(\mathrm{a}_{2}\right)$ fails occurs when $a$ is assumed to be a positive constant, $A>0$, in $[z-\eta, z)$, and a negative constant, $-B<0$, in $(z, z+\eta]$, for some small $\eta>0$. In such case, owing to Theorem 1.1, all solutions in $\mathscr{C}_{\lambda_{0}}^{+}$develop a singularity when they become sufficiently large. On the contrary, all solutions are regular if, for instance, the weight $a$ is differentiable at the nodal point $z$.
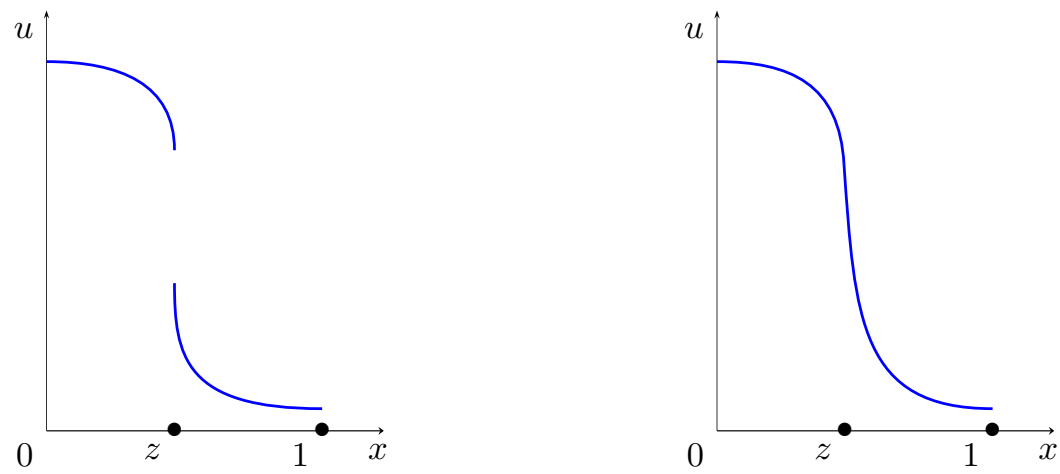

Figure 1. Profiles of the solutions within $\mathscr{S}^{+}$having sufficiently large norms: on the left the case where $\left(\mathrm{a}_{2}\right)$ fails, on the right the case where $\left(\mathrm{a}_{2}\right)$ holds.

According to $\left[27\right.$, Th. 1.4], if in addition to $\left(\mathrm{f}_{1}\right)$ we assume that $f \in \mathcal{C}^{2}(\mathbb{R})$, then, thanks to the local bifurcation theorem of [8], in a neighborhood of $\left(\lambda_{0}, 0\right)$ the component $\mathscr{C}_{\lambda_{0}}^{+}$consists of a $\mathcal{C}^{1}$-curve, $\left(\lambda(s), s\left(\varphi_{0}+v(s)\right)\right)$, for $s$ close to 0 , such that $v(0)=0, \lambda(0)=\lambda_{0}$, and

with

$$
\lambda^{\prime}(0)=-\lambda_{0} \frac{f^{\prime \prime}(0)}{2} \frac{\int_{0}^{1} a(x) \varphi_{0}^{3}(x) d x}{\int_{0}^{1} a(x) \varphi_{0}^{2}(x) d x}
$$

$$
\int_{0}^{1} a(x) \varphi_{0}^{2}(x) d x>0 \text { and } \int_{0}^{1} a(x) \varphi_{0}^{3}(x) d x>0 .
$$

Therefore, $\mathscr{C}_{\lambda_{0}}^{+}$bifurcates transcritically from $\left(\lambda_{0}, 0\right)$ if $f^{\prime \prime}(0) \neq 0$. Precisely, this bifurcation is supercritical if $f^{\prime \prime}(0)<0$, while it is subcritical if $f^{\prime \prime}(0)>0$. Should it occurs that $f^{\prime \prime}(0)=0$ and $f \in \mathcal{C}^{3}(\mathbb{R})$, like for the special choices made in (1.2), then, by [27, Eq. (4.2)], $\lambda^{\prime \prime}(0)<0$. Therefore, the bifurcation of $\mathscr{C}_{\lambda_{0}}^{+}$in this case also is subcritical.

By modulating the constant $h$ in $\left(\mathrm{f}_{1}\right)$, the value of $\lambda$ where $\mathscr{C}_{\lambda_{0}}^{+}$bifurcates from infinity, $\lambda_{\infty}$, can be at any side of $\lambda_{0}$; hence, any of the components $\mathscr{C}_{\lambda_{0}}^{+}$plotted in Figure 1 are admissible. Indeed, the bifurcation value from infinity

satisfies

$$
\lambda_{\infty}=\lambda_{\infty}(h)=\frac{1}{h \int_{0}^{z} a(x) d x}
$$

$$
\lim _{h \rightarrow+\infty} \lambda_{\infty}(h)=0, \quad \lim _{h \rightarrow 0^{+}} \lambda_{\infty}(h)=+\infty .
$$

This clearly produces the existence of multiple solutions for $\lambda>\lambda_{0}$, if $\lambda_{\infty} \leq \lambda_{0}$ and the bifurcation from $\lambda_{0}$ is supercritical, as it is displayed by the first two plots of Figure 1, as well as for $\lambda<\lambda_{0}$, if $\lambda_{\infty} \geq \lambda_{0}$ and the bifurcation from $\lambda_{0}$ is subcritical. Figure 2 illustrates the special case when $\mathscr{C}_{\lambda_{0}}^{+}$bifurcates supercritically from $\lambda_{0}$. Similar bifurcation diagrams occur if the bifurcation from zero is subcritical.

The structure of this paper is the following. Section 2 contains the main non-existence result, Section 3 analyzes the bifurcation of the large solutions from infinity, Section 4 ascertains the exact limiting profiles of the large positive solutions of (1.1) and, finally, Section 5 delivers the proof of Theorem 1.1. 

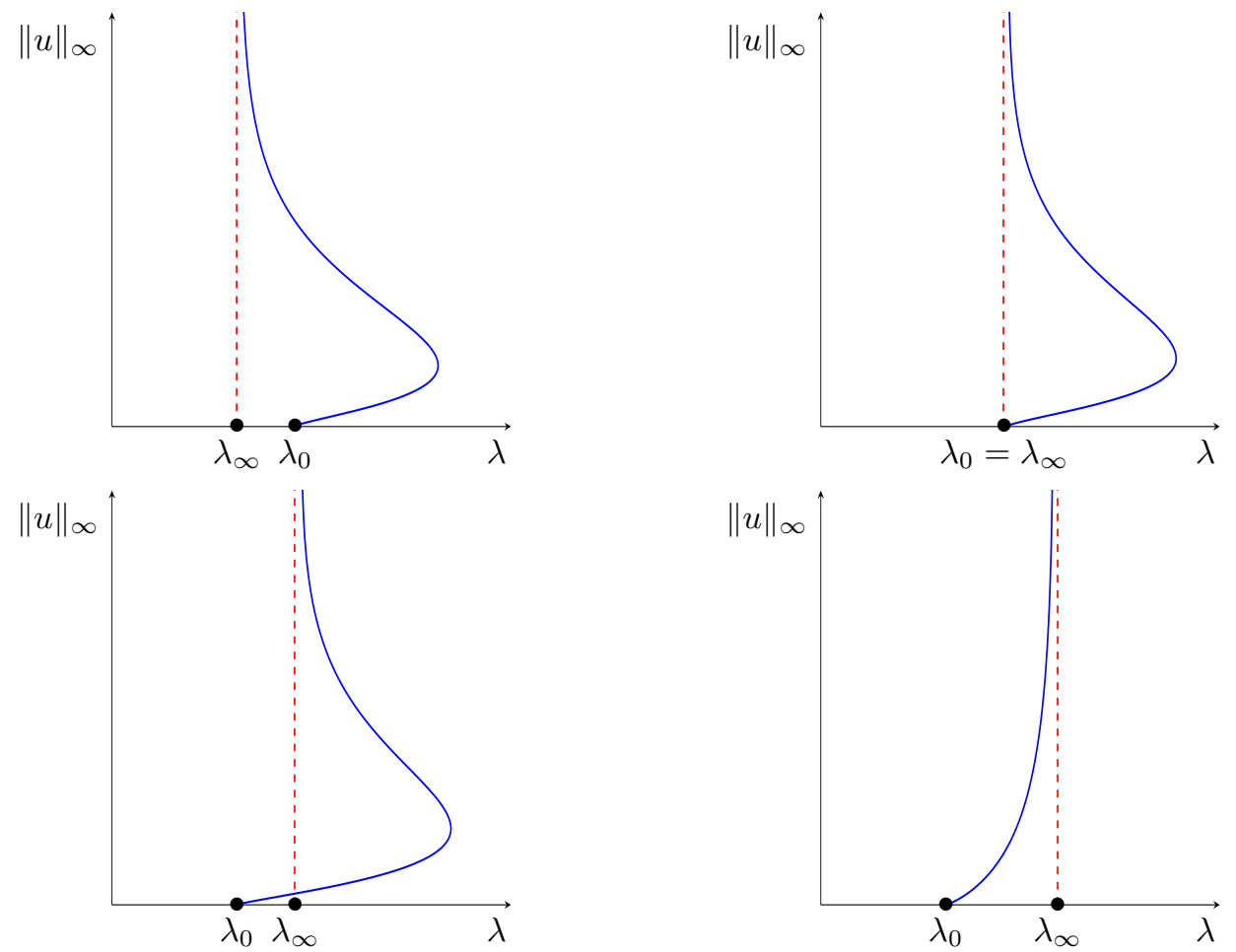

Figure 2. The global components $\mathscr{C}_{\lambda_{0}}^{+}$bifurcating from zero at $\lambda_{0}$ and from infinity at $\lambda_{\infty}$. The case where supercritical bifurcation occurs at $\lambda_{0}$ is displayed.

\section{NON-EXISTENCE OF POSITIVE SOLUTIONS FOR SMALL AND LARGE $\lambda>0$}

The main result of this section can be stated as follows.

Theorem 2.1. Assume $\left(\mathrm{a}_{1}\right)$ and $\left(\mathrm{f}_{1}\right)$. Then, the problem (1.1) has no positive solutions both for sufficiently small and for sufficiently large $\lambda>0$.

Proof. In order to prove that (1.1) has no positive solutions for all small $\lambda>0$, we will argue by contradiction assuming that (1.1) admits a sequence of positive solutions, $\left(\left(\lambda_{n}, u_{n}\right)\right)_{n \geq 1}$, such that $\lambda_{n}>0$ for all $n \geq 1$ and

$$
\lim _{n \rightarrow+\infty} \lambda_{n}=0 .
$$

Integrating the differential equation of $(1.1)$ in $(0, z)$, we get for all $n \geq 1$

$$
\frac{-u_{n}^{\prime}(z)}{\sqrt{1+\left(u_{n}^{\prime}(z)\right)^{2}}}=\lambda_{n} \int_{0}^{z} a(x) f\left(u_{n}(x)\right) d x .
$$

Thus, since $a$ and $f$ are bounded, letting $n \rightarrow+\infty$ in (2.2) it follows from (2.1) that

Hence, by (1.3), we find that

$$
\lim _{n \rightarrow+\infty} \frac{-u_{n}^{\prime}(z)}{\sqrt{1+\left(u_{n}^{\prime}(z)\right)^{2}}}=0 \text {. }
$$

$$
0=\lim _{n \rightarrow+\infty} u_{n}^{\prime}(z)=\lim _{n \rightarrow+\infty}\left\|u^{\prime}\right\|_{L^{\infty}(0,1)}
$$

and, consequently, $\left(\lambda_{n}, u_{n}\right)$ is a regular solution of (1.1) for sufficiently large $n$. Further, possibly passing to a subsequence, relabeled by $n$, there exists $L \in[0,+\infty]$ such that

$$
\lim _{n \rightarrow+\infty} u_{n}(0)=L .
$$


According to (2.3), from the identity

$$
u_{n}(x)=u_{n}(0)+\int_{0}^{x} u_{n}^{\prime}(t) d t \quad \text { for } x \in[0,1]
$$

it is easily seen that

$$
\lim _{n \rightarrow+\infty} u_{n}=L \quad \text { uniformly on }[0,1] .
$$

Next, we will distinguish between three different cases according to the value of $L$.

Suppose that $L=0$. Then, setting

$$
v_{n}=\frac{u_{n}}{\left\|u_{n}\right\|_{L^{\infty}(0,1)}} \quad \text { and } \quad A_{n}=a \frac{f\left(u_{n}\right)}{u_{n}}\left[1+\left(u_{n}^{\prime}\right)^{2}\right]^{\frac{3}{2}},
$$

we find that

$$
\left\{\begin{array}{l}
-v_{n}^{\prime \prime}=\lambda_{n} A_{n}(x) v_{n} \quad \text { in }(0,1) \\
v_{n}^{\prime}(0)=v_{n}^{\prime}(0)=0
\end{array}\right.
$$

Since

$$
\left\|v_{n}\right\|_{L^{\infty}(0,1)}=1 \text { for all } n \geq 1
$$

and, due to $\left(\mathrm{a}_{1}\right),\left(\mathrm{f}_{1}\right)$ and $(2.3)$, we have that

$$
\lim _{n \rightarrow+\infty}\left\|A_{n}-a\right\|_{L^{\infty}(0,1)}=0
$$

letting $n \rightarrow+\infty$ in (2.4), it follows from (2.1) that

$$
\lim _{n \rightarrow+\infty}\left\|v_{n}^{\prime \prime}\right\|_{L^{\infty}(0,1)}=0 .
$$

Thus, since

we also have that

$$
v_{n}^{\prime}(x)=v_{n}^{\prime}(0)+\int_{0}^{x} v_{n}^{\prime \prime}(t) d t=\int_{0}^{x} v_{n}^{\prime \prime}(t) d t \quad \text { for } x \in[0,1],
$$

$$
\lim _{n \rightarrow+\infty}\left\|v_{n}^{\prime}\right\|_{L^{\infty}(0,1)}=0 .
$$

Therefore, possibly passing to a further subsequence, still labeled by $n$, we find that

$$
\lim _{n \rightarrow+\infty} v_{n}(x)=1 \quad \text { uniformly in } x \in[0,1] \text {. }
$$

Finally, dividing (2.4) by $\lambda_{n}$ and integrating on $[0,1]$ yields

$$
0=\int_{0}^{1} A_{n}(x) v_{n}(x) d x \quad \text { for all } n \geq 1 .
$$

Thus, we conclude that

which is a contradiction

$$
0=\lim _{n \rightarrow+\infty} \int_{0}^{1} A_{n}(x) v_{n}(x) d x=\int_{0}^{1} a(x) d x<0,
$$

Now, suppose that $L \in(0,+\infty)$. Then, integrating (1.1) on $[0,1]$, we see that

$$
0=-\lambda_{n}^{-1} \int_{0}^{1}\left(\frac{u_{n}^{\prime}(x)}{\sqrt{1+\left(u_{n}^{\prime}(x)\right)^{2}}}\right)^{\prime} d x=\int_{0}^{1} a(x) f\left(u_{n}(x)\right) d x
$$

for all $n \geq 1$. Thus, letting $n \rightarrow+\infty$, we find that

$$
0=f(L) \int_{0}^{1} a(x) d x<0
$$

which is a contradiction, because $f(L)>0$ and $\int_{0}^{1} a(x) d x<0$.

Finally, suppose that $L=+\infty$. Then, the same argument yields

$$
0=h \int_{0}^{1} a(x) d x<0
$$


which is as well impossible. This concludes the proof of the non-existence of positive solutions for sufficiently small $\lambda>0$.

To prove the non-existence for sufficiently large $\lambda>0$, we will also argue by contradiction. So, assume that there is a sequence of positive solutions of $(1.1),\left(\left(\lambda_{n}, u_{n}\right)\right)_{n \geq 1}$, with

$$
\lim _{n \rightarrow+\infty} \lambda_{n}=+\infty \text {. }
$$

Then, it follows from (2.2) and (2.5) that

$$
\lim _{n \rightarrow+\infty} \int_{0}^{z} a(x) f\left(u_{n}(x)\right) d x=\lim _{n \rightarrow+\infty}\left(\frac{1}{\lambda_{n}} \frac{-u_{n}^{\prime}(z)}{\sqrt{1+\left(u_{n}^{\prime}(z)\right)^{2}}}\right)=0
$$

and hence, along some subsequence, relabeled by $n$, it becomes apparent that

$$
\lim _{n \rightarrow+\infty}\left(a(x) f\left(u_{n}(x)\right)\right)=0 \quad \text { for a.e. } x \in[0, z] .
$$

Thus, owing to $\left(\mathrm{a}_{1}\right)$ and $\left(\mathrm{f}_{1}\right)$, we deduce that

$$
\lim _{n \rightarrow+\infty} u_{n}(x)=0 \text { for a.e. } x \in[0, z] .
$$

Therefore, since $u_{n}$ is decreasing,

$$
\lim _{n \rightarrow+\infty} u_{n}(x)=0 \text { for all } x \in(0,1] .
$$

Moreover, possibly passing to a further subsequence, there exists $L \in[0,+\infty]$ such that

$$
\lim _{n \rightarrow+\infty} u_{n}(0)=L .
$$

Subsequently we will distinguish between two different cases. First, suppose that $L \in(0,+\infty]$. Then, the concavity of $u_{n}$ on $[0, z)$ implies that, for any given $y \in(0, z)$,

$$
u_{n}^{\prime}(y) \leq \frac{u_{n}(y)-u_{n}(0)}{y}<0
$$

and hence, (2.6) and (2.7) provide us with

$$
\lim _{n \rightarrow+\infty} u_{n}^{\prime}(y) \leq \lim _{n \rightarrow+\infty} \frac{u_{n}(y)-u_{n}(0)}{y}= \begin{cases}-\frac{L}{y} & \text { if } L \in(0,+\infty) \\ -\infty & \text { if } L=+\infty\end{cases}
$$

By the concavity, we also have that

$$
u_{n}(x) \leq u_{n}(y)+u_{n}^{\prime}(y)(x-y) \quad \text { for all } x \in(y, z) .
$$

Note that the right hand side of (2.9) vanishes at

$$
x_{n}=y-\frac{u_{n}(y)}{u_{n}^{\prime}(y)} .
$$

Moreover, thanks to (2.6) and (2.8), it becomes apparent that

$$
\lim _{n \rightarrow+\infty} x_{n}=y<z .
$$

Thus, for sufficiently large $n$, we find that $x_{n}<z$. As this forces $u_{n}$ to vanish in $(0, z)$, which is impossible. The proof is completed in this case.

Lastly, suppose that $L=0$. Then, since $u_{n}(0)=\|u\|_{L^{\infty}(0,1)}$,

$$
\lim _{n \rightarrow+\infty} u_{n}(x)=0 \quad \text { uniformly in } x \in[0,1] .
$$

Thus, by $\left(\mathrm{f}_{1}\right)$, we can infer that, for sufficiently large $n$,

$$
f\left(u_{n}(x)\right) \geq \frac{1}{2} u_{n}(x) \text { for all } x \in[0,1] .
$$

Hence, it follows from the differential equation of (1.1) that

$$
-u_{n}^{\prime \prime}(x)=\lambda_{n} a(x) f\left(u_{n}(x)\right)\left(1+\left(u_{n}^{\prime}(x)\right)^{2}\right)^{\frac{3}{2}} \geq \frac{1}{2} \lambda_{n} a(x) u_{n}(x) \quad \text { for a.e. } x \in[0, z] \text {. }
$$


Subsequently, we denote by $\mu_{1}>0$ the unique principal eigenvalue of the weighted eigenvalue problem

$$
\left\{\begin{aligned}
-\varphi^{\prime \prime} & =\mu a(x) \varphi \text { in }(0, z) \\
\varphi(0) & =\varphi(z)=0
\end{aligned}\right.
$$

and consider any positive eigenfunction, $\varphi_{1}>0$, associated to $\mu_{1}$. We have that $\mu_{1}>0$, because $a(x)>0$ for all $x \in(0, z)$ (cf. [23, Ch. 9]), and, moreover, $\varphi_{1}(x)>0$ for all $x \in(0, z), \varphi_{1}^{\prime}(0)>0$ and $\varphi_{1}^{\prime}(z)<0$. Thus, multiplying $(2.10)$ by $\varphi_{1}$ and integrating by parts twice in $(0, z)$ yield

$$
\begin{aligned}
\frac{1}{2} \lambda_{n} & \int_{0}^{z} a(x) u_{n}(x) \varphi_{1}(x) d x \leq-\int_{0}^{z} u_{n}^{\prime \prime}(x) \varphi_{1}(x) d x \\
& =u_{n}^{\prime}(0) \varphi_{1}(0)-u_{n}^{\prime}(z) \varphi_{1}(z)+\int_{0}^{z} u_{n}^{\prime}(x) \varphi_{1}^{\prime}(x) d x=\int_{0}^{z} u_{n}^{\prime}(x) \varphi_{1}^{\prime}(x) d x \\
& =u_{n}(z) \varphi_{1}^{\prime}(z)-u_{n}(0) \varphi_{1}^{\prime}(0)-\int_{0}^{z} u_{n}(x) \varphi_{1}^{\prime \prime}(x) d x<-\int_{0}^{z} \varphi_{1}^{\prime \prime}(x) u_{n}(x) d x
\end{aligned}
$$

Therefore, according to (2.11), we see that

$$
\frac{1}{2} \lambda_{n} \int_{0}^{z} a(x) u_{n}(x) \varphi_{1}(x) d x<\mu_{1} \int_{0}^{z} a(x) \varphi_{1}(x) u_{n}(x) d x
$$

for all $n \geq 1$. Since

$$
\int_{0}^{z} a(x) \varphi_{1}(x) u_{n}(x) d x>0
$$

it follows from (2.12) that $\lambda_{n}<2 \mu_{1}$, which contradicts (2.5) and ends the proof.

According to Theorem 2.1, the values of the parameter $\lambda$ defined by

$$
\lambda_{*}=\inf \left\{\lambda>0 \mid(\lambda, u) \in \mathscr{S}^{+}\right\}, \quad \lambda^{*}=\sup \left\{\lambda>0 \mid(\lambda, u) \in \mathscr{S}^{+}\right\},
$$

satisfy

$$
0<\lambda_{*} \leq \lambda^{*}<+\infty
$$

Therefore, by Theorem 2.1,

$$
\lambda \in\left[\lambda_{*}, \lambda^{*}\right] \subset(0,+\infty) \text { if }(\lambda, u) \in \mathscr{S}^{+} \backslash(\{0\} \times[0,+\infty)) .
$$

\section{BIFURCATION OF POSITIVE SOLUTIONS FROM ZERO AND FROM INFINITY}

According to [24, Th. 5.13], the component of $\mathscr{S}^{+}$bifurcating from $(\lambda, u)=\left(\lambda_{0}, 0\right)$, denoted in this paper by $\mathscr{C}_{\lambda_{0}}^{+}$, is unbounded in $\mathbb{R} \times L^{\infty}(0,1)$. Moreover, Theorem 2.1 implies that $\lambda \in\left[\lambda_{*}, \lambda^{*}\right]$ if $(\lambda, u) \in \mathscr{C}_{\lambda_{0}}^{+}$. Therefore, we conclude that $\operatorname{proj}_{\mathrm{L}^{\infty}(0,1)} \mathscr{C}_{\lambda_{0}}^{+}$and, hence, $\operatorname{proj}_{\mathrm{L}^{\infty}(0,1)}\left(\mathscr{S}^{+} \backslash(\{0\} \times[0,+\infty))\right)$ must be unbounded. Accordingly, from any unbounded sequence of positive solutions in $\mathscr{S}^{+} \backslash(\{0\} \times[0,+\infty))$ one can extract a subsequence $\left(\left(\lambda_{n}, u_{n}\right)\right)_{n \geq 1}$ and find a number $\lambda_{\infty}$ such that

$$
\lim _{n \rightarrow+\infty} \lambda_{n}=\lambda_{\infty} \in\left[\lambda_{*}, \lambda^{*}\right] \text { and } \lim _{n \rightarrow \infty}\left\|u_{n}\right\|_{L^{\infty}(0,1)}=+\infty .
$$

In particular, since $u_{n}(0)=\left\|u_{n}\right\|_{L^{\infty}(0,1)}$, we have that

$$
\lim _{n \rightarrow+\infty} u_{n}(0)=+\infty \text {. }
$$

Throughout this section we will assume that (3.2) holds and the precise value of $\lambda_{\infty}$ will be ascertained by showing that

$$
\lambda_{\infty}=\left(h \int_{0}^{z} a(x) d x\right)^{-1} .
$$

The independence of $\lambda_{\infty}$ from the particular sequence $\left(\left(\lambda_{n}, u_{n}\right)\right)_{n \geq 1}$, satisfying (3.1), proves that $\lambda_{\infty}$ is the only bifurcation point from infinity of $\mathscr{S}^{+} \backslash(\{0\} \times[0,+\infty))$. In particular, $\mathscr{C}_{\lambda_{0}}^{+}$is a connected component of $\mathscr{S}^{+}$bifurcating from zero and from infinity.

In the sequel $\left(\left(\lambda_{n}, u_{n}\right)\right)_{n \geq 1}$ stands for any sequence satisfying (3.1), for some number $\lambda_{\infty}$. We begin with the next result of technical nature. 
Lemma 3.1. Assume $\left(\mathrm{a}_{1}\right)$, $\left(\mathrm{f}_{1}\right)$ and (3.2). Then, the following hold:

$$
\limsup _{n \rightarrow+\infty} u_{n}(1)<+\infty \text { and } \lim _{n \rightarrow+\infty} u_{n}^{\prime}(z)=-\infty .
$$

Proof. To prove the first conclusion, we will argue by contradiction assuming that, along some subsequence, also labeled by $n$, one has that

$$
\lim _{n \rightarrow+\infty} u_{n}(1)=+\infty
$$

and hence

$$
\lim _{n \rightarrow+\infty} u_{n}(x)=+\infty \quad \text { uniformly in } x \in[0,1] .
$$

Thus, taking $\phi=1$ as the test function in the definition of bounded variation solution and letting $n \rightarrow+\infty$, assumption $\left(\mathrm{f}_{1}\right)$ yields

$$
\begin{aligned}
h \int_{0}^{z} a(x) d x & \left.=\lim _{n \rightarrow+\infty} \int_{0}^{z} a(x) f\left(u_{n}(x)\right)\right) d x \\
& =-\lim _{n \rightarrow+\infty} \int_{z}^{1} a(x) f\left(u_{n}(x)\right) d x=-h \int_{1}^{z} a(x) d x .
\end{aligned}
$$

and hence, $\int_{0}^{1} a(x) d x=0$, contradicting $\left(\mathrm{a}_{1}\right)$. Therefore, the first assertion holds. The second assertion, which requires to be proven only for regular solutions, follows readily from (3.2) and the first assertion of (3.3) taking into account that

$$
-u_{n}^{\prime}(z)=\left\|u_{n}^{\prime}\right\|_{L^{\infty}(0,1)}
$$

This ends the proof.

The next result provides us with uniform a priori bounds for the first and second derivatives of the solutions $u_{n}$ on any interval of the form $[0, z-\eta], \eta>0$.

Proposition 3.1. Assume $\left(\mathrm{a}_{1}\right),\left(\mathrm{f}_{1}\right)$ and $(3.2)$. Then, for every $\eta \in(0, z)$, there exists a constant $C>0$ such that

$$
\max \left\{\left\|u_{n}^{\prime}\right\|_{L^{\infty}(0, z-\eta)},\left\|u_{n}^{\prime \prime}\right\|_{L^{\infty}(0, z-\eta)}\right\} \leq C
$$

Proof. Fix $\eta \in(0, z)$ and, arguing by contradiction, suppose that there is a subsequence of $\left(\left(\lambda_{n}, u_{n}\right)\right)_{n \geq 1}$, still labeled by $n$, such that

$$
\lim _{n \rightarrow+\infty} u_{n}^{\prime}(z-\eta)=-\infty .
$$

The monotonicity of $u_{n}^{\prime}$ on $[0, z)$ implies that

$$
\lim _{n \rightarrow+\infty} u_{n}^{\prime}(x)=-\infty \text { for all } x \in[z-\eta, z] .
$$

Thus, integrating the differential equation on $(z-\eta, z)$ and letting $n \rightarrow+\infty$ yield

$$
\begin{aligned}
0 & =\lim _{n \rightarrow+\infty}\left(-\frac{u_{n}^{\prime}(z)}{\sqrt{1+\left(u_{n}^{\prime}(z)\right)^{2}}}+\frac{u_{n}^{\prime}(z-\eta)}{\sqrt{1+\left(u_{n}^{\prime}(z-\eta)\right)^{2}}}\right) \\
& =\lim _{n \rightarrow+\infty}\left(\lambda_{n} \int_{z-\eta}^{z} a(x) f\left(u_{n}(x)\right) d x\right) .
\end{aligned}
$$

Hence, thanks to (2.13), we infer that

$$
\lim _{n \rightarrow+\infty} \int_{z-\eta}^{z} a(x) f\left(u_{n}(x)\right) d x=0 .
$$

Since $a(x)>0$ for a.e. $x \in(0, z)$, this implies that, for a subsequence relabeled by $n$,

$$
\lim _{n \rightarrow+\infty} f\left(u_{n}(x)\right)=0 \text { for a.e. } x \in(z-\eta, z) .
$$

Then, using $\left(\mathrm{f}_{1}\right)$ and taking into account that $u_{n}$ is decreasing, we find that

$$
\lim _{n \rightarrow+\infty} u_{n}(x)=0 \text { for all } x \in(z-\eta, 1] .
$$


Fix $y \in(z-\eta, z)$. Then, since

$$
\lim _{n \rightarrow+\infty} u_{n}(y)=0 \text { and } \lim _{n \rightarrow+\infty} u_{n}^{\prime}(y)=-\infty,
$$

the concavity of $u_{n}$ on $[0, z)$ entails that

$$
\lim _{n \rightarrow+\infty} u_{n}\left(z^{-}\right) \leq \lim _{n \rightarrow+\infty}\left(u_{n}(y)+u_{n}^{\prime}(y)(z-y)\right)=-\infty,
$$

which is impossible, because $u_{n}(x) \geq 0$ for all $x \in[0,1]$. Therefore, there exists a constant $C>0$ such that

$$
\left\|u_{n}^{\prime}\right\|_{L^{\infty}(0, z-\eta)} \leq C
$$

for all $n \geq 1$. By $\left(f_{1}\right)$, the estimate for the second derivatives follows readily by rewriting the differential equation in the form

$$
-u_{n}^{\prime \prime}=\lambda_{n} a f\left(u_{n}\right)\left(1+\left(u_{n}^{\prime}\right)^{2}\right)^{\frac{3}{2}} \quad \text { in }[0, z)
$$

and taking into account that $\lambda_{n} \in\left[\lambda_{*}, \lambda^{*}\right]$ for all $n \geq 1$, because of Theorem 2.1. This ends the proof.

The next results provides us with the pointwise behavior of the solutions $u_{n}$, as $n \rightarrow+\infty$, on the interval $[0, z)$.

Theorem 3.1. Assume $\left(\mathrm{a}_{1}\right)$, $\left(\mathrm{f}_{1}\right)$ and (3.2). Then, for every $\eta \in(0, z)$, one has

$$
\lim _{n \rightarrow+\infty} u_{n}(x)=+\infty \quad \text { uniformly in } x \in[0, z-\eta]
$$

and, in particular,

$$
\lim _{n \rightarrow+\infty} u_{n}(x)=+\infty \quad \text { for all } x \in[0, z) .
$$

Proof. Taking into account that, for every $n \geq 1$ and $x \in[0, z)$,

$$
u_{n}(x)=u_{n}(0)+\int_{0}^{x} u_{n}^{\prime}(t) d t
$$

it is easily seen that (3.5) follows from (3.2) and (3.4). As (3.4) holds for arbitrarily small $\eta>0$, (3.6) holds too. This ends the proof.

The next result establishes the exact value of $\lambda_{\infty}$ in (3.1).

Theorem 3.2. Assume $\left(\mathrm{a}_{1}\right)$, $\left(\mathrm{f}_{1}\right)$ and (3.2). Then, one has

$$
\lim _{n \rightarrow+\infty} \lambda_{n}=\lambda_{\infty}=\left(h \int_{0}^{z} a(x) d x\right)^{-1} .
$$

Proof. By (2.2), we have that

$$
\lambda_{n}=\frac{-u_{n}^{\prime}(z)}{\sqrt{1+\left(u_{n}^{\prime}(z)\right)^{2}}}\left(\int_{0}^{z} a(x) f\left(u_{n}(x)\right) d x\right)^{-1}
$$

for all $n \geq 1$. Thus, letting $n \rightarrow+\infty$ in the previous identity, (3.7) follows from (3.3) and (3.6) through the dominated convergence theorem, because $f$ is bounded.

\section{Sharp limiting Behavior of the Large Positive solutions}

Thanks to the analysis done in Section 3 , we already know that, for any sequence $\left(\left(\lambda_{n}, u_{n}\right)\right)_{n \geq 1}$ in $\mathscr{S}^{+} \backslash$ $(\{0\} \times[0,+\infty))$, satisfying (3.2), the condition (3.7) holds, and, moreover,

$$
\lim _{n \rightarrow+\infty} u_{n}(x)=+\infty \text { for all } x \in[0, z) .
$$

This section aims to establish the precise asymptotic behavior of the solutions $u_{n}$, for large $n$.

The next result provides us with the exact profile of the derivatives, $u_{n}^{\prime}$, of the solutions on the interval $[0, z)$. 
Theorem 4.1. Assume $\left(\mathrm{a}_{1}\right),\left(\mathrm{f}_{1}\right)$ and (3.2). Then, for every $\eta \in(0, z)$, one has

$$
\lim _{n \rightarrow+\infty} u_{n}^{\prime}(x)=\frac{-\int_{0}^{x} a(t) d t}{\sqrt{\left(\int_{0}^{z} a(t) d t\right)^{2}-\left(\int_{0}^{x} a(t) d t\right)^{2}}} \quad \text { uniformly in } x \in[0, z-\eta] \text {, }
$$

and, in particular,

$$
\lim _{n \rightarrow+\infty} u_{n}^{\prime}(x)=\frac{-\int_{0}^{x} a(t) d t}{\sqrt{\left(\int_{0}^{z} a(t) d t\right)^{2}-\left(\int_{0}^{x} a(t) d t\right)^{2}}} \quad \text { for all } x \in[0, z) .
$$

Proof. Let denote by $\psi: \mathbb{R} \rightarrow(-1,1)$ the map defined by

$$
\psi(s)=\frac{s}{\sqrt{1+s^{2}}} .
$$

The function $\psi$ is invertible with inverse $\psi^{-1}:(-1,1) \rightarrow \mathbb{R}$ defined by

$$
\psi^{-1}(t)=\frac{t}{\sqrt{1-t^{2}}}
$$

Fix $\eta \in(0, z)$ and pick any $x \in[0, z-\eta]$. Then, integrating the differential equation on $(0, x)$ yields, for all $n \geq 1$,

or, equivalently,

$$
\psi\left(-u_{n}^{\prime}(x)\right)=\lambda_{n} \int_{0}^{x} a(t) f\left(u_{n}(t)\right) d t
$$

$$
u_{n}^{\prime}(x)=\psi^{-1}\left(-\lambda_{n} \int_{0}^{x} a(t) f\left(u_{n}(t)\right) d t\right) .
$$

Therefore, letting $n \rightarrow+\infty$ in this identity and taking into account Theorems 3.1 and 3.2, the dominated convergence theorem and the continuity of $\psi^{-1}$ implies that, for every $x \in[0, z-\eta]$,

$$
\lim _{n \rightarrow+\infty} u_{n}^{\prime}(x)=\psi^{-1}\left(-\lambda_{\infty} h \int_{0}^{x} a(t) d t\right)=\frac{-\int_{0}^{x} a(t) d t}{\sqrt{\left(\int_{0}^{z} a(t) d t\right)^{2}-\left(\int_{0}^{x} a(t) d t\right)^{2}}} .
$$

The uniform convergence on $[0, z-\eta]$ is a direct consequence of Proposition 3.1 and the Ascoli-Arzelà theorem. The proof is complete.

The next result complements Proposition 3.1 on the interval $[z+\eta, 1]$.

Proposition 4.1. Assume $\left(\mathrm{a}_{1}\right),\left(\mathrm{f}_{1}\right)$ and $(3.2)$. Then, for every $\eta \in(0,1-z)$, there exists a constant $C>0$ such that

$$
\max \left\{\left\|u_{n}^{\prime}\right\|_{L^{\infty}(z+\eta, 1)},\left\|u_{n}^{\prime \prime}\right\|_{L^{\infty}(z+\eta, 1)}\right\} \leq C .
$$

Proof. The proof basically follows the same patterns as the one of Proposition 3.1. Fix $\eta \in(0,1-z)$ and, arguing by contradiction, suppose that there is some subsequence of $\left(\left(\lambda_{n}, u_{n}\right)\right)_{n \geq 1}$, relabeled by $n$, such that

$$
\lim _{n \rightarrow+\infty} u_{n}^{\prime}(z+\eta)=-\infty .
$$

Then, by the monotonicity of $u_{n}$ on $[z, 1]$, this actually implies that

$$
\lim _{n \rightarrow+\infty} u_{n}^{\prime}(x)=-\infty \text { for all } x \in[z, z+\eta] .
$$

Thus, integrating the differential equation in $(1.1)$ on $(z, z+\eta)$ and letting $n \rightarrow+\infty$, we find that

$$
\lim _{n \rightarrow+\infty}\left(\lambda_{n} \int_{z}^{z+\eta} a(x) f\left(u_{n}(x)\right) d x\right)=0 .
$$

Consequently, since, by $(2.13)$, we already know that $\lambda_{n} \in\left[\lambda_{*}, \lambda^{*}\right]$, it follows that

$$
\lim _{n \rightarrow+\infty} \int_{z}^{z+\eta} a(x) f\left(u_{n}(x)\right) d x=0 .
$$


Hence, by $\left(\mathrm{a}_{1}\right)$, along some subsequence, still labeled by $n$, we find that

$$
\lim _{n \rightarrow+\infty} f\left(u_{n}(x)\right)=0 \quad \text { for a.e. } x \in[z, z+\eta] .
$$

Therefore, by the monotonicity of $f\left(u_{n}\right)$, this implies that

$$
\lim _{n \rightarrow+\infty} f\left(u_{n}(x)\right)=0 \quad \text { for all } x \in(z, 1] .
$$

Lastly, using $\phi=1$ as a test function in the definition of bounded variation solution, it follows from Theorem 3.1 and the dominated convergence theorem that

$$
0=-\lim _{n \rightarrow+\infty} \int_{z}^{1} a(x) f\left(u_{n}(x)\right) d x=\lim _{n \rightarrow+\infty} \int_{0}^{z} a(x) f\left(u_{n}(x)\right) d x=h \int_{0}^{z} a(x) d x>0,
$$

which is impossible. This contradiction provides us with the uniform a priori bounds for $u_{n}^{\prime}$ on $[z+\eta, 1]$. The uniform bounds on $u_{n}^{\prime \prime}$ can be obtained as in the proof of Proposition 3.1; therefore, the technical details are omitted here.

The next result complements Theorem 3.1 by providing us with the limiting behavior of the solutions $u_{n}$, as $n \rightarrow+\infty$, on the interval $(z, 1]$.

Proposition 4.2. Assume $\left(\mathrm{a}_{1}\right)$, $\left(\mathrm{f}_{1}\right)$ and (3.2). Then, there exists a subsequence, $\left(\left(\lambda_{n_{k}}, u_{n_{k}}\right)\right)_{k \geq 1}$ of $\left(\left(\lambda_{n}, u_{n}\right)\right)_{n \geq 1}$ for which the pointwise limit

$$
u_{\infty}(x)=\lim _{k \rightarrow+\infty} u_{n_{k}}(x), \quad \text { for all } x \in(z, 1],
$$

is well-defined, satisfies $u_{\infty} \in W_{\mathrm{loc}}^{2, \infty}(z, 1]$ and solves the problem

$$
\left\{\begin{array}{l}
-\left(\frac{u^{\prime}}{\sqrt{1+\left(u^{\prime}\right)^{2}}}\right)^{\prime}=\lambda_{\infty} a(x) f(u) \quad \text { in }(z, 1), \\
u^{\prime}(z)=-\infty, u^{\prime}(1)=0 .
\end{array}\right.
$$

Moreover, the following hold:

and either

$$
\begin{gathered}
u_{\infty}(x)>0 \quad \text { for all } x \in(z, 1], \\
\lim _{k \rightarrow+\infty} u_{n_{k}}=u_{\infty} \quad \text { in } W_{\text {loc }}^{2, \infty}(z, 1]
\end{gathered}
$$

$$
u_{\infty}\left(z^{+}\right)<+\infty \text { if } \int_{z}^{1}\left(\int_{x}^{z} a(t) d t\right)^{-\frac{1}{2}} d x<+\infty
$$

or

$$
u_{\infty}\left(z^{+}\right)=+\infty \quad \text { if } \int_{z}^{1}\left(\int_{x}^{z} a(t) d t\right)^{-\frac{1}{2}} d x=+\infty .
$$

Proof. Fix any $\eta \in(0,1-z)$. By Proposition 4.1 , there exists a subsequence, $\left(\left(\lambda_{n_{k}}, u_{n_{k}}\right)\right)_{k \geq 1}$, of $\left(\left(\lambda_{n}, u_{n}\right)\right)_{n \geq 1}$ and a function $u_{\infty, \eta} \in \mathcal{C}^{1}[z+\eta, 1]$ such that

$$
\lim _{k \rightarrow+\infty} u_{n_{k}}=u_{\infty, \eta} \quad \text { in } \mathcal{C}^{1}[z+\eta, 1]
$$

Thus, letting $n \rightarrow+\infty$ in the differential equations

$$
-u_{n_{k}}^{\prime \prime}(x)=\lambda_{n_{k}} a(x) f\left(u_{n_{k}}(x)\right)\left(1+\left(u_{n_{k}}^{\prime}(x)\right)^{2}\right)^{\frac{3}{2}} \quad \text { for a.e. in } x \in(z+\eta, 1),
$$

it becomes apparent, by Theorem 3.2, that actually $u_{\infty, \eta} \in W^{2, \infty}(z+\eta, 1)$ and it satisfies

$$
-u_{\infty, \eta}^{\prime \prime}(x)=\lambda_{\infty} a(x) f\left(u_{\infty, \eta}(x)\right)\left(1+\left(u_{\infty, \eta}^{\prime}(x)\right)^{2}\right)^{\frac{3}{2}} \text { for a.e. in } x \in(z+\eta, 1) .
$$

Now, consider any sequence $\left(\eta_{j}\right)_{j \geq 1}$ such that $\eta_{j} \in(0,1-z)$, for all $j \geq 1$, and

$$
\lim _{j \rightarrow+\infty} \eta_{j}=0
$$


and consider, for every $j \geq 1$, the associated $u_{\infty, \eta_{j}}$. By a diagonal argument, we can extract a further subsequence of $\left(\left(\lambda_{n_{k}}, u_{n_{k}}\right)\right)_{k \geq 1}$, still labeled by $k$, and a function $u_{\infty} \in W_{\text {loc }}^{2, \infty}(z, 1]$ such that

$$
\lim _{k \rightarrow+\infty} u_{n_{k}}=u_{\infty} \quad \text { in } W_{\text {loc }}^{2, \infty}(z, 1]
$$

and

or, in other words,

$$
-u_{\infty}^{\prime \prime}(x)=\lambda_{\infty} a(x) f\left(u_{\infty}(x)\right)\left(1+\left(u_{\infty}^{\prime}(x)\right)^{2}\right)^{\frac{3}{2}} \quad \text { for a.e. in } x \in(z, 1),
$$

$$
-\left(\frac{u_{\infty}^{\prime}(x)}{\sqrt{1+\left(u_{\infty}^{\prime}(x)\right)^{2}}}\right)^{\prime}=\lambda_{\infty} a(x) f\left(u_{\infty}(x)\right) \text { for a.e. } x \in(z, 1) .
$$

By the construction, it follows easily from (4.3) that

$$
u_{\infty}(x) \geq 0 \text { for all } x \in(z, 1] \text { and } u_{\infty}^{\prime}(1)=0 .
$$

Thus, $u_{\infty}$ is convex and decreasing in $(z, 1]$. Moreover, since $f(0)=0$, the uniqueness of the solution of the associated Cauchy problem entails that $u_{\infty}(1)>0$ and hence

$$
u_{\infty}(x)>0 \text { for all } x \in(z, 1] .
$$

Let us show that

$$
u_{\infty}^{\prime}\left(z^{+}\right)=-\infty, \quad \text { if } u_{\infty}\left(z^{+}\right)<+\infty
$$

Indeed, if $u_{\infty}^{\prime}\left(z^{+}\right) \in \mathbb{R}$, then, by continuous dependence of the solutions of the associated Cauchy problems on the initial conditions and on the parameters, would imply that

$$
\lim _{k \rightarrow+\infty} u_{n_{k}}(z)=u_{\infty}^{\prime}(z) \in \mathbb{R},
$$

which contradicts Lemma 3.1.

Moreover, by the convexity of $u_{\infty}$, we also must have that

$$
u_{\infty}^{\prime}\left(z^{+}\right)=-\infty, \quad \text { if } u_{\infty}\left(z^{+}\right)=+\infty .
$$

Consequently, in any case we can conclude that

$$
u_{\infty}^{\prime}\left(z^{+}\right)=-\infty .
$$

To complete the proof, it remains to characterize the finiteness of $u_{\infty}\left(z^{+}\right)$in terms of the integrability properties of the weight function $a$. Integrating (4.2) in $(z, x)$, with $x \in(z, 1)$, and using (4.4) yields

and hence

$$
0<\frac{-u_{\infty}^{\prime}(x)}{\sqrt{1+\left(u_{\infty}^{\prime}(x)\right)^{2}}}=1+\lambda_{\infty} \int_{z}^{x} a(t) f\left(u_{\infty}(t)\right) d t<1
$$

$$
-u_{\infty}^{\prime}(x)=\frac{1+\lambda_{\infty} \int_{z}^{x} a(t) f\left(u_{\infty}(t)\right) d t}{\sqrt{2+\lambda_{\infty} \int_{z}^{x} a(t) f\left(u_{\infty}(t)\right) d t}} \cdot \frac{1}{\sqrt{\lambda_{\infty} \int_{x}^{z} a(t) f\left(u_{\infty}(t)\right) d t}} .
$$

Therefore, for sufficiently small $\eta>0$, the following estimates hold

$$
\frac{1}{2} \frac{1}{\sqrt{\lambda_{\infty} h}}\left(\int_{x}^{z} a(t) d t\right)^{-\frac{1}{2}} \leq-u_{\infty}^{\prime}(x) \leq \frac{1}{\sqrt{\lambda_{\infty} f\left(u_{\infty}(1)\right)}}\left(\int_{x}^{z} a(t) d t\right)^{-\frac{1}{2}}
$$

for every $x \in(z, z+\eta)$. Pick $x \in(z, z+\eta)$. Then, integrating (4.5) on $(x, z+\eta)$ yields

$$
\begin{aligned}
\frac{1}{2} \frac{1}{\sqrt{\lambda_{\infty} h}} \int_{x}^{z+\eta}\left(\int_{t}^{z} a(s) d s\right)^{-\frac{1}{2}} d t & \leq u_{\infty}(x)-u_{\infty}(z+\eta) \\
& \leq \frac{1}{\sqrt{\lambda_{\infty} f\left(u_{\infty}(1)\right)}} \int_{x}^{z+\eta}\left(\int_{t}^{z} a(s) d s\right)^{-\frac{1}{2}} d t
\end{aligned}
$$

Suppose

$$
\int_{z}^{1}\left(\int_{x}^{z} a(t) d t\right)^{-\frac{1}{2}} d x<+\infty
$$


Then, letting $x \rightarrow z^{+}$in the previous inequalities provides us with

$$
u_{\infty}\left(z^{+}\right) \leq u_{\infty}(z+\eta)+\frac{1}{\sqrt{\lambda_{\infty} f\left(u_{\infty}(1)\right)}} \int_{z}^{z+\eta}\left(\int_{t}^{z} a(s) d s\right)^{-\frac{1}{2}} d t<+\infty .
$$

Similarly, in case

$$
\int_{z}^{1}\left(\int_{x}^{z} a(t) d t\right)^{-\frac{1}{2}} d x=+\infty
$$

we find that

$$
u_{\infty}\left(z^{+}\right) \geq u_{\infty}(z+\eta)+\frac{1}{2} \frac{1}{\sqrt{\lambda_{\infty} h}} \int_{z}^{z+\eta}\left(\int_{t}^{z} a(s) d s\right)^{-\frac{1}{2}} d t=+\infty .
$$

The proof is complete.

The next result describes the ultimate behavior of the solutions $u_{n}$ at the endpoints of the interval $[z, 1]$.

Proposition 4.3. Assume $\left(\mathrm{a}_{1}\right),\left(\mathrm{f}_{1}\right)$ and (3.2). Then, one has

$$
\liminf _{n \rightarrow+\infty} u_{n}(1)>0
$$

and

$$
\limsup _{n \rightarrow+\infty} u_{n}\left(z^{+}\right)<+\infty, \quad \text { if } \int_{z}^{1}\left(\int_{x}^{z} a(t) d t\right)^{-\frac{1}{2}} d x<+\infty
$$

whereas

$$
\limsup _{n \rightarrow+\infty} u_{n}\left(z^{+}\right)=+\infty, \quad \text { if } \int_{z}^{1}\left(\int_{x}^{z} a(t) d t\right)^{-\frac{1}{2}} d x=+\infty .
$$

Proof. To prove (4.6), we proceed by contradiction assuming that there exists a subsequence, $\left(\left(\lambda_{n_{k}}, u_{n_{k}}\right)\right)_{k \geq 1}$, of $\left(\left(\lambda_{n}, u_{n}\right)\right)_{n \geq 1}$ such that

$$
\lim _{k \rightarrow+\infty} u_{n_{k}}(1)=0 .
$$

Applying Proposition 4.2 to this subsequence yields

$$
\limsup _{k \rightarrow+\infty} u_{n_{k}}(1)>0
$$

which contradicts (4.9) and ends the proof of (4.6).

Next, we will prove (4.7). Integrating the differential equation of (1.1), we obtain, for every $n \geq 1$ and $x \in(z, 1)$,

thus, setting

$$
0<\frac{-u_{n}^{\prime}(x)}{\sqrt{1+\left(u_{n}^{\prime}(x)\right)^{2}}}=\frac{-u_{n}^{\prime}(z)}{\sqrt{1+\left(u_{n}^{\prime}(z)\right)^{2}}}-\lambda_{n} \int_{x}^{z} a(t) f\left(u_{n}(t)\right) d t<1
$$

$$
\varphi_{n}(x, z)=\frac{-u_{n}^{\prime}(z)}{\sqrt{1+\left(u_{n}^{\prime}(z)\right)^{2}}}-\lambda_{n} \int_{x}^{z} a(t) f\left(u_{n}(t)\right) d t,
$$

we have that

$$
-u_{n}^{\prime}(x)=\frac{\varphi_{n}(x, z)}{\sqrt{1+\varphi_{n}(x, z)}} \cdot \frac{1}{\sqrt{1-\varphi_{n}(x, z)}} .
$$

Thanks to Proposition 4.3, Lemma 3.1 and Theorem 3.2, respectively, we can find $M>0$ and $n_{0} \in \mathbb{N}$ such that, for every $n \geq n_{0}$,

$$
\frac{M}{2} \leq u_{n}(1) \leq M \quad \text { and } \quad \frac{\lambda_{\infty}}{2} \leq \lambda_{n} .
$$

By (4.10), for every $n \geq 1$ and $x \in(z, 1)$, we have that

$$
\frac{\varphi_{n}(x, z)}{\sqrt{1+\varphi_{n}(x, z)}}<\varphi_{n}(x, z)<1 .
$$


Thus, due to (4.11) and (4.10), we find that

$$
\begin{aligned}
-u_{n}^{\prime}(x) & \leq \frac{1}{\sqrt{1-\varphi_{n}(x, z)}}=\frac{1}{\sqrt{1+\frac{u_{n}^{\prime}(z)}{\sqrt{1+\left(u_{n}^{\prime}(z)\right)^{2}}}+\lambda_{n} \int_{x}^{z} a(t) f\left(u_{n}(t)\right) d t}} \\
& \leq \frac{1}{\sqrt{\lambda_{n} \int_{x}^{z} a(t) f\left(u_{n}(t)\right) d t}} \leq \frac{1}{\sqrt{\lambda_{n} f\left(u_{n}(1)\right)}}\left(\int_{x}^{z} a(t) d t\right)^{-\frac{1}{2}} .
\end{aligned}
$$

So, by (4.12), we get

$$
-u_{n}^{\prime}(x) \leq \sqrt{\frac{2}{\lambda_{\infty} f(M / 2)}}\left(\int_{x}^{z} a(t) d t\right)^{-\frac{1}{2}}
$$

and therefore

$$
u_{n}\left(z^{+}\right)=u_{n}(1)-\int_{z}^{1} u_{n}^{\prime}(x) d x \leq M+\sqrt{\frac{2}{\lambda_{\infty} f(M / 2)}} \int_{z}^{1}\left(\int_{x}^{z} a(t) d t\right)^{-\frac{1}{2}} d x
$$

which implies (4.7).

To prove (4.8), we argue by contradiction assuming that there is a subsequence, $\left(\left(\lambda_{n_{k}}, u_{n_{k}}\right)\right)_{k \geq 1}$, of $\left(\left(\lambda_{n}, u_{n}\right)\right)_{n \geq 1}$ such that

$$
\limsup _{k \rightarrow+\infty} u_{n_{k}}\left(z^{+}\right)<+\infty .
$$

This implies the existence of a constant $C>0$ such that

$$
\sup _{(z, 1)} u_{n_{k}}=u_{n_{k}}\left(z^{+}\right) \leq C
$$

for all $k \geq 1$. Applying Proposition 4.2 to this subsequence yields

$$
\sup _{(z, 1)} u_{\infty} \leq C,
$$

as $u_{\infty}$ is the pointwise limit in $(z, 1)$ of $\left(u_{n_{k}}\right)_{k \geq 1}$. Since we are assuming

$$
\int_{z}^{1}\left(\int_{x}^{z} a(t) d t\right)^{-\frac{1}{2}} d x=+\infty
$$

(4.14) contradicts the last assertion of Proposition 4.2 , because $u_{\infty}\left(z^{+}\right)=+\infty$ in such case. The proof is complete.

The next result establishes the uniqueness of the positive solution of the problem (4.2).

Proposition 4.4. For every $\lambda>0$, the problem (4.2) has at most one solution $u \in W_{\mathrm{loc}}^{2, \infty}(z, 1]$ with $u(x)>0$ for all $x \in(z, 1]$.

Proof. First, suppose that $u$ and $v$ are two solutions of (4.2) satisfying $u(x)>0, v(x)>0$ for all $x \in(z, 1]$ and

$$
u\left(z^{+}\right)<+\infty, \quad v\left(z^{+}\right)<+\infty
$$

Set $w=u-v$. As

$$
u^{\prime}\left(z^{+}\right)=v^{\prime}\left(z^{+}\right)=-\infty
$$

and hence

$$
\frac{u^{\prime}\left(z^{+}\right)}{\sqrt{1+\left(u^{\prime}\left(z^{+}\right)\right)^{2}}}=-1=\frac{v^{\prime}\left(z^{+}\right)}{\sqrt{1+\left(v^{\prime}\left(z^{+}\right)\right)^{2}}},
$$


we easily get from (4.2)

$$
\begin{aligned}
\lambda \int_{z}^{1} a(x) f(u(x)) w(x) d x & =-\int_{z}^{1}\left(\frac{u^{\prime}(x)}{\sqrt{1+\left(u^{\prime}(x)\right)^{2}}}\right)^{\prime} w(x) d x \\
& =\frac{u^{\prime}\left(z^{+}\right)}{\sqrt{1+\left(u^{\prime}\left(z^{+}\right)\right)^{2}}} w\left(z^{+}\right)+\int_{z}^{1} \frac{u^{\prime}(x) w^{\prime}(x)}{\sqrt{1+\left(u^{\prime}(x)\right)^{2}}} d x \\
& =-w\left(z^{+}\right)+\int_{z}^{1} \frac{u^{\prime}(x) w^{\prime}(x)}{\sqrt{1+\left(u^{\prime}(x)\right)^{2}}} d x
\end{aligned}
$$

and

$$
\begin{aligned}
\lambda \int_{z}^{1} a(x) f(v(x)) w(x) d x & =-\int_{z}^{1}\left(\frac{v^{\prime}(x)}{\sqrt{1+\left(v^{\prime}(x)\right)^{2}}}\right)^{\prime} w(x) d x \\
& =\frac{v^{\prime}\left(z^{+}\right)}{\sqrt{1+\left(v^{\prime}\left(z^{+}\right)\right)^{2}}} w\left(z^{+}\right)+\int_{z}^{1} \frac{v^{\prime}(x) w^{\prime}(x)}{\sqrt{1+\left(v^{\prime}(x)\right)^{2}}} d x \\
& =-w\left(z^{+}\right)+\int_{z}^{1} \frac{v^{\prime}(x) w^{\prime}(x)}{\sqrt{1+\left(v^{\prime}\right)(x)^{2}}} d x
\end{aligned}
$$

Subtracting the above identities yields

$$
\begin{aligned}
\int_{z}^{1}\left(\frac{u^{\prime}(x)}{\sqrt{1+\left(u^{\prime}(x)\right)^{2}}}-\frac{v^{\prime}(x)}{\sqrt{1+\left(v^{\prime}(x)\right)^{2}}}\right) & \left(u^{\prime}(x)-v^{\prime}(x)\right) d x \\
& =\lambda \int_{z}^{1} a(x)(f(u(x))-f(v(x)))(u(x)-v(x)) d x .
\end{aligned}
$$

By using the monotonicity of the functions $f$ and $\psi$, with $\psi$ defined by (4.1), and taking also into account that $\lambda>0$ and $a(x)<0$ for a.e. $x \in(z, 1)$, we find hat

$$
\int_{z}^{1} a(x)(f(u(x))-f(v(x)))(u(x)-v(x)) d x=0
$$

and, therefore, $u=v$.

Now, suppose that $u$ and $v$ are two solutions of (4.2) satisfying $u(x)>0, v(x)>0$ for all $x \in(z, 1]$ and

$$
u\left(z^{+}\right)=v\left(z^{+}\right)=+\infty .
$$

The proof that $u=v$ in this case is divided into two steps.

Step 1. The solutions $u$ and $v$ are ordered, i.e., either $u(x) \leq v(x)$ for all $x \in(z, 1)$, or $v(x) \leq u(x)$ for all $x \in(z, 1)$. Since $u^{\prime}(1)=v^{\prime}(1)=0$, if $u(1)=v(1)$, by the uniqueness of solution for the associated Cauchy problem, we conclude that $u=v$. So, suppose that, e.g., $u(1)<v(1)$ and that there exists $y \in(z, 1)$ such that

$$
u(y)=v(y) \text { and } u(x)<v(x) \text { for all } x \in(y, 1] .
$$

Set $w=u-v$. As $w(y)=0$, we get

$$
\begin{aligned}
& \lambda \int_{y}^{1} a(x) f(u(x)) w(x) d x=-\int_{y}^{1}\left(\frac{u^{\prime}(x)}{\sqrt{1+\left(u^{\prime}(x)\right)^{2}}}\right)^{\prime} w(x) d x=\int_{y}^{1} \frac{u^{\prime}(x) w^{\prime}(x)}{\sqrt{1+\left(u^{\prime}(x)\right)^{2}}} d x \\
& \lambda \int_{y}^{1} a(x) f(v(x)) w(x) d x=-\int_{y}^{1}\left(\frac{v^{\prime}(x)}{\sqrt{1+\left(v^{\prime}(x)\right)^{2}}}\right)^{\prime} w(x) d x=\int_{y}^{1} \frac{v^{\prime}(x) w^{\prime}(x)}{\sqrt{1+\left(v^{\prime}(x)\right)^{2}}} d x .
\end{aligned}
$$


Therefore, subtracting these identities and arguing as above shows that

$$
\begin{gathered}
\int_{y}^{1}\left(\frac{u^{\prime}(x)}{\sqrt{1+\left(u^{\prime}(x)\right)^{2}}}-\frac{v^{\prime}(x)}{\sqrt{1+\left(v^{\prime}(x)\right)^{2}}}\right)\left(u^{\prime}(x)-v^{\prime}(x)\right) d x \\
=\lambda \int_{y}^{1} a(x)(f(u(x))-f(v(x)))(u(x)-v(x)) d x=0 .
\end{gathered}
$$

Consequently, we have that $u(x)=v(x)$ for all $x \in(y, 1]$, which is impossible. This ends the proof of Step 1 .

Step 2. The solutions $u$ and $v$ satisfy $v=u$. Assume that, e.g., $v(x) \geq u(x)$ for all $x \in(z, 1]$ and define, for every $k>0$, the function

Then we get, for a.e. $x \in(z, 1)$,

$$
w_{k}=\min \{v-u, k\}
$$

$$
\begin{aligned}
\left(\frac{u^{\prime}(x)}{\sqrt{1+\left(u^{\prime}(x)\right)^{2}}}\right. & \left.-\frac{v^{\prime}(x)}{\sqrt{1+\left(v^{\prime}(x)\right)^{2}}}\right) w_{k}^{\prime}(x) \\
& = \begin{cases}\left(\frac{u^{\prime}(x)}{\sqrt{1+\left(u^{\prime}(x)\right)^{2}}}-\frac{v^{\prime}(x)}{\sqrt{1+\left(v^{\prime}(x)\right)^{2}}}\right)\left(v^{\prime}(x)-u^{\prime}(x)\right) \leq 0 & \text { if } v(x)-u(x) \leq k, \\
0 & \text { if } v(x)-u(x)>k,\end{cases}
\end{aligned}
$$

and thus

$$
\left(\frac{u^{\prime}(x)}{\sqrt{1+\left(u^{\prime}(x)\right)^{2}}}-\frac{v^{\prime}(x)}{\sqrt{1+\left(v^{\prime}(x)\right)^{2}}}\right) w_{k}^{\prime}(x) \leq 0 .
$$

On the other hand, for every $y \in(z, 1)$, we have that

$$
\begin{aligned}
\lambda \int_{y}^{1} a(x)(f(u(x))-f(v(x))) w_{k}(x) d x & =-\int_{y}^{1}\left(\frac{u^{\prime}(x)}{\sqrt{1+\left(u^{\prime}(x)\right)^{2}}}-\frac{v^{\prime}}{\sqrt{1+\left(v^{\prime}(x)\right)^{2}}}\right) w_{k}(x) d x \\
= & \left(\frac{u^{\prime}(y)}{\sqrt{1+\left(u^{\prime}(y)\right)^{2}}}-\frac{v^{\prime}(y)}{\sqrt{1+\left(v^{\prime}(y)\right)^{2}}}\right) w_{k}(y) \\
& +\int_{y}^{1}\left(\frac{u^{\prime}(x)}{\sqrt{1+\left(u^{\prime}(x)\right)^{2}}}-\frac{v^{\prime}(x)}{\sqrt{1+\left(v^{\prime}(x)\right)^{2}}}\right) w_{k}^{\prime}(x) d x
\end{aligned}
$$

Thus, by (4.16), we find that

$$
\lambda \int_{y}^{1} a(x)(f(u(x))-f(v(x))) w_{k}(x) d x \leq\left(\frac{u^{\prime}(y)}{\sqrt{1+\left(u^{\prime}(y)\right)^{2}}}-\frac{v^{\prime}(y)}{\sqrt{1+\left(v^{\prime}(y)\right)^{2}}}\right) w_{k}(y)
$$

for all $y \in(z, 1)$. So, letting $y \rightarrow z^{+}$in this inequality, condition (4.15) and the boundedness of $w_{k}$ imply that

$$
\lambda \int_{z}^{1} a(x)(f(u(x))-f(v(x))) w_{k}(x) d x \leq 0
$$

But, since $\lambda>0$ and, for a.e. $x \in(z, 1), a(x)<0, f(u(x)) \leq f(v(x)), w_{k}(x) \geq 0$, we conclude that

$$
\int_{z}^{1} a(x)(f(u(x))-f(v(x))) w_{k}(x) d x=0 .
$$

Hence, we infer that, for a.e. $x \in(z, 1)$,

$$
a(x)(f(u(x))-f(v(x))) w_{k}(x)=0 \quad \text { for a.e. } x \in(z, 1) .
$$

and then

Letting $k \rightarrow+\infty$ yields

$$
(f(v(x))-f(u(x))) w_{k}(x)=0 \quad \text { for all } x \in(z, 1] .
$$

$$
(f(v(x))-f(u(x)))(v(x)-u(x))=0 \quad \text { for all } x \in(z, 1] . .
$$


The monotonicity of $f$ entails $u=v$. The proof is complete.

Finally, thanks to Propositions 4.2, 4.3 , 4.4, we can describe the exact asymptotic behavior of the solutions $u_{n}$ in the interval $(z, 1]$.

Theorem 4.2. Assume $\left(\mathrm{a}_{1}\right),\left(\mathrm{f}_{1}\right)$ and $(3.2)$. Then, the whole sequence, $\left(\left(\lambda_{n}, u_{n}\right)\right)_{n \geq 1}$ converges to $\left(\lambda_{\infty}, u_{\infty}\right)$ in $\mathbb{R} \times W_{\text {loc }}^{2, \infty}(z, 1]$ as $n \rightarrow+\infty$, where $\lambda_{\infty}$ is given by (3.7) and $u_{\infty}$ is the unique solution of (4.2) satisfying $u_{\infty}(x)>0$ for all $x \in(z, 1]$.

\section{Proof of Theorem 2.1}

From $\left[24\right.$, Th. 5.1.3] it is easily inferred the existence of two connected components, $\mathscr{C}_{0}^{+}$and $\mathscr{C}_{\lambda_{0}}^{+}$, satisfying the following conditions:

(1) $\mathscr{C}_{0}^{+}$and $\mathscr{C}_{\lambda_{0}}^{+}$are unbounded in $\mathbb{R} \times L^{\infty}(0,1)$;

(2) $\mathscr{C}_{0}^{+}$and $\mathscr{C}_{\lambda_{0}}^{+}$are closed and connected subsets of $\mathbb{R} \times B V(0,1)$ with $B V(0,1)$ endowed with the topology of the strict convergence;

(3) $(0,0) \in \mathscr{C}_{0}^{+}$and $\left(\lambda_{0}, 0\right) \in \mathscr{C}_{\lambda_{0}}^{+}$

(4) $\{(0, r): r \geq 0\} \subseteq \mathscr{C}_{0}^{+}$;

(5) ess inf $u>0$ if $(\lambda, u) \in \mathscr{C}_{0}^{+} \cup \mathscr{C}_{\lambda_{0}}^{+}$with $u \neq 0$;

(6) $\lambda=\lambda_{0}$ if $(\lambda, 0) \in \mathscr{C}_{0}^{+} \cup \mathscr{C}_{\lambda_{0}}^{+}$with $\lambda>0$;

(7) either $\mathscr{C}_{0}^{+} \cap \mathscr{C}_{\lambda_{0}}^{+}=\emptyset$, or $(0,0) \in \mathscr{C}_{\lambda_{0}}^{+}$and $\left(\lambda_{0}, 0\right) \in \mathscr{C}_{0}^{+}$, and, in such case, $\mathscr{C}_{0}^{+}=\mathscr{C}_{\lambda_{0}}^{+}$;

(8) there is a neighborhood, $\mathcal{V}$, of $(0,0)$ in $\mathbb{R} \times L^{\infty}(0,1)$ such that $\mathscr{C}_{0}^{+} \cap \mathcal{V}$ consists of regular solutions;

(9) there is a neighborhood, $\mathcal{U}$, of $\left(\lambda_{0}, 0\right)$ in $\mathbb{R} \times L^{\infty}(0,1)$ such that $\mathscr{C}_{\lambda_{0}}^{+} \cap \mathcal{U}$ consists of regular solutions. Combining these properties with the theory developed in the previous sections allows us to verify all the conclusions of Theorem 1.1.

Indeed, from conditions (3), (4), (6), (7) and Theorem 2.1 it readily follows that

$$
\mathscr{C}_{0}^{+}=\{(0, r): r \geq 0\} \quad \text { and } \quad \mathscr{C}_{0}^{+} \cap \mathscr{C}_{\lambda_{0}}^{+}=\emptyset .
$$

Thus, (a) and (b) hold. The conclusions (c) and (d) are consequences of (1) and Theorem 2.1. Theorem 3.2 yields (e). The conclusion (f) follows from Theorems 3.1 and 4.1, for what concerns the behavior on the interval $[0, z)$, and from Theorem 4.2 and Proposition 4.2, for what concerns the behavior on the interval $(z, 1]$. While assertion (g) is precisely $(9)$.

In order to prove $(\mathrm{h})$, we proceed by contradiction. So, assume that $\left(\mathrm{a}_{2}\right)$ holds and that $(\lambda, u) \in \mathscr{S}^{+}$, with $\lambda>0$, is a singular solution. Then, thanks to (1.4), for any $x \in(0,1) \backslash\{z\}$, integrating the differential equation in $(x, z)$ yields

$$
0<\frac{-u^{\prime}(x)}{\sqrt{1+\left(u^{\prime}(x)\right)^{2}}}=1-\lambda \int_{x}^{z} a(t) f(u(t)) d t<1
$$

and hence

$$
-u^{\prime}(x)=\frac{1-\lambda \int_{x}^{z} a(t) f(u(t)) d t}{\sqrt{2-\lambda \int_{x}^{z} a(t) f(u(t)) d t}} \cdot \frac{1}{\sqrt{\lambda \int_{x}^{z} a(t) f(u(t)) d t}} .
$$

So, for sufficiently small $\eta>0$ and every $x \in[z-\eta, z+\eta] \backslash\{z\}$, we have that

$$
-u^{\prime}(x) \geq \frac{1}{2 \sqrt{\lambda h}}\left(\int_{x}^{z} a(t) d t\right)^{-\frac{1}{2}} \text {. }
$$

Under condition $\left(\mathrm{a}_{2}\right)$, this estimate entails that either $u^{\prime} \notin L^{1}(0, z)$, or $u^{\prime} \notin L^{1}(z, 1)$, which contradicts the fact that

$$
u \in W^{1,1}(0, z) \cap W^{1,1}(z, 1) .
$$

Therefore, assuming $\left(\mathrm{a}_{2}\right)$, the set $\mathscr{S}^{+}$consists of regular solutions, as claimed by assertion $(\mathrm{h})$.

Lastly, we will prove the conclusion (h). More generally, we will show that all the solutions of $\mathscr{S}^{+}$with sufficiently large $L^{\infty}$-norm must develop singularities if $\left(a_{2}\right)$ fails. We will argue by contradiction assuming the existence of a sequence of regular solutions, $\left(\left(\lambda_{n}, u_{n}\right)\right)_{n \geq 1}$, in $\mathscr{S}^{+}$such that

$$
\lim _{n \rightarrow+\infty}\left\|u_{n}\right\|_{L^{\infty}}=+\infty
$$


Then, by Theorem 3.2, we also have that

$$
\lim _{n \rightarrow+\infty} \lambda_{n}=\lambda_{\infty}
$$

As we are assuming that

$$
\int_{z}^{1}\left(\int_{x}^{z} a(t) d t\right)^{-\frac{1}{2}} d x<+\infty
$$

it follows from Proposition 4.3 that

$$
0<\liminf _{n \rightarrow \infty} u_{n}(z) \leq \limsup _{n \rightarrow+\infty} u_{n}(z)<+\infty .
$$

Thus, there exist $n_{0} \in \mathbb{N}$ and $M>0$ such that, for every $n \geq n_{0}$,

$$
\frac{M}{2} \leq u_{n}(z) \leq M \quad \text { and } \quad \lambda_{n} \geq \frac{\lambda_{\infty}}{2} .
$$

Since these estimates coincide with (4.12), reasoning as in the proof of Proposition 4.3 it becomes apparent that (4.13) holds, i.e.,

$$
-u_{n}^{\prime}(x) \leq \sqrt{\frac{2}{\lambda_{\infty} f(M / 2)}}\left(\int_{x}^{z} a(t) d t\right)^{-\frac{1}{2}},
$$

and hence

$$
\begin{aligned}
u_{n}(0) & =u_{n}(z)-\int_{0}^{z} u_{n}^{\prime}(x) d x \\
& \leq M+\sqrt{\frac{2}{\lambda_{\infty} f(M / 2)}} \int_{0}^{z}\left(\int_{x}^{z} a(t) d t\right)^{-\frac{1}{2}} d x=C<+\infty,
\end{aligned}
$$

as we are assuming that ( $\left.\mathrm{a}_{2}\right)$ fails. This implies that, for all $n \geq n_{0}$,

$$
\left\|u_{n}\right\|_{L^{\infty}}=u_{n}(0) \leq C
$$

which contradicts (5.1) and ends the proof of Theorem 1.1.

\section{REFERENCES}

[1] L. Ambrosio, N. Fusco and D. Pallara, Functions of Bounded Variation and Free Discontinuity Problems, Clarendon Press, Oxford, 2000

[2] G. Anzellotti, The Euler equation for functionals with linear growth, Trans. Amer. Math. Soc. 290 (1985), 483-501.

[3] E. Bombieri, E. De Giorgi and M. Miranda, Una maggiorazione a priori relativa alle ipersuperfici minimali non parametriche, Arch. Ration. Mech. Anal. 32 (1969), 255-267.

[4] M. Burns and M. Grinfeld, Steady state solutions of a bi-stable quasi-linear equation with saturating flux, European $J$. Appl. Math. 22 (2011), 317-331.

[5] P. Concus and R. Finn, On a class of capillary surfaces, J. Anal. Math. 23 (1970), 65-70.

[6] C. Corsato, C. De Coster, N. Flora and P. Omari, Radial solutions of the Dirichlet problem for a class of quasilinear elliptic equations arising in optometry, Nonlinear Anal. 181 (2019), 9-23.

[7] C. Corsato, C. De Coster and P. Omari, The Dirichlet problem for a prescribed anisotropic mean curvature equation: existence, uniqueness and regularity of solutions, J. Differential Equations 260 (2016), 4572-4618.

[8] M. G. Crandall and P. H. Rabinowitz, Bifurcation from simple eigenvalues, J. Funct. Anal. 8 (1971), 321-340.

[9] M. Emmer, Esistenza, unicità e regolarità nelle superfici di equilibrio nei capillari, Ann. Univ. Ferrara 18 (1973), 79-94.

[10] R. Finn, The sessile liquid drop. I. Symmetric case, Pacific J. Math. 88 (1980), 541-587.

[11] R. Finn, Equilibrium Capillary Surfaces, Springer, New York, 1986.

[12] R. Finn, On the equations of capillarity, J. Math. Fluid Mech. 3 (2001), 139-151.

[13] R. Finn, Capillarity problems for compressible fluids, Mem. Differential Equations Math. Phys. 33 (2004), 47-55.

[14] R. Finn and G. Luli, On the capillary problem for compressible fluids, J. Math. Fluid Mech. 9 (2007), 87-103.

[15] C. Gerhardt, Boundary value problems for surfaces of prescribed mean curvature, J. Math. Pures Appl. 58 (1979), 75-109.

[16] C. Gerhardt, Global $C^{1,1}$-regularity for solutions of quasilinear variational inequalities, Arch. Ration. Mech. Anal. 89 (1985), 83-92

[17] E. Giusti, Boundary value problems for non-parametric surfaces of prescribed mean curvature, Ann. Sc. Norm. Super. Pisa Cl. Sci. 3 (1976), 501-548.

[18] E. Giusti, Minimal Surfaces and Functions of Bounded Variations, Birkhäuser, Basel, 1984. 
[19] E. Gonzalez, U. Massari and I. Tamanini, Existence and regularity for the problem of a pendent liquid drop, Pacific J. Math. 88 (1980), 399-420.

[20] G. Huisken, Capillary surfaces over obstacles, Pacific J. Math. 117 (1985), 121-141.

[21] A. Kurganov and P. Rosenau, On reaction processes with saturating diffusion, Nonlinearity 19 (2006), 171-193.

[22] O. A. Ladyzhenskaya and N. N. Ural'tseva, Local estimates for gradients of solutions of non-uniformly elliptic and parabolic equations, Comm. Pure Appl. Math. 23 (1970), 677-703.

[23] J. López-Gómez, Linear Second Order Elliptic Operators, World Scientific, Singapore, 2013.

[24] J. López-Gómez and P. Omari, Global components of positive bounded variation solutions of a one-dimensional indefinite quasilinear Neumann problem, Adv. Nonlinear Studies 19 (2019), 437-473.

[25] J. López-Gómez and P. Omari, Characterizing the formation of singularities in a superlinear indefinite problem related to the mean curvature operator, J. Differential Equations (2020), https://doi.org/10.1016/j.jde.2020.01.015.

[26] J. López-Gómez, P. Omari and S. Rivetti, Positive solutions of one-dimensional indefinite capillarity-type problems J. Differential Equations 262 (2017), 2335-2392.

[27] J. López-Gómez, P. Omari and S. Rivetti, Bifurcation of positive solutions for a one-dimensional indefinite quasilinear Neumann problem Nonlinear Anal. 155 (2017), 1-51.

[28] P. Rosenau, Free energy functionals at the high gradient limit, Phys. Rev. A 41 (1990), 2227-2230.

[29] J. Serrin, The problem of Dirichlet for quasilinear elliptic differential equations with many independent variables, Philos. Trans. Roy. Soc. London 264 (1969) 413-496.

[30] R. Temam, Solutions généralisées de certaines équations du type hypersurfaces minima, Arch. Ration. Mech. Anal. 44 $(1971 / 72), 121-156$.

Universidad Complutense de Madrid, Departamento de Matemática Aplicada, Plaza de Ciencias 3,28040 Madrid, SPAIN

E-mail address: julian@mat.ucm.es

Università degli Studi di Trieste, Dipartimento di Matematica e Geoscienze, Via A. Valerio $12 / 1,34127$ Trieste, ITALY

E-mail address: omari@units.it 